\title{
Trajectories for the Wave Function of the Universe from a Simple Detector Model
}

\author{
J.J.HALLIWELL
}

Theory Group Blackett Laboratory Imperial

College London SW7 2BZ UK

PreprintImperial/TP/99-0/37. Revised version. November, 2000

\begin{abstract}
Inspired by Mott's (1929) analysis of particle tracks in a cloud chamber, we consider a simple model for quantum cosmology which includes, in the total Hamiltonian, model detectors registering whether or not the system, at any stage in its entire history, passes through a series of regions in configuration space. We thus derive a variety of well-defined formulas for the probabilities for trajectories associated with the solutions to the Wheeler-DeWitt equation. The probability distribution is peaked about classical trajectories in configuration space. The "measured" wave functions still satisfy the Wheeler-DeWitt equation, except for small corrections due to the disturbance of the measuring device. With modified boundary conditions, the measurement amplitudes essentially agree with an earlier result of Hartle derived on rather different grounds. In the special case where the system is a collection of harmonic oscillators, the interpretation of the results is aided by the introduction of "timeless" coherent states - eigenstates of the Hamiltonian which are concentrated about entire classical trajectories.
\end{abstract}




\section{INTRODUCTION}

The focus of attention in quantum cosmology is the Wheeler-DeWitt equation,

$$
H \Psi=0
$$

Here, the wave function $\Psi$ is a functional of the gravitational and matter fields on a threesurface, and it describes the quantum state of a closed cosmological model [1]. The most striking and conceptually problematic aspect of this equation is that it does not involve time explicitly, severely complicating efforts to extract predictions from it $[2,3]$. Amongst the many attempts to understand this feature, one is to claim that "time", and indeed entire histories of the universe, are already contained amongst the arguments of the wave function, hence no time label is required $[4,5]$. Whilst these claims seem to be true at some level in simple models of quantum cosmology, it presents us with the interesting challenge of reformulating standard quantum theory without the explicit use of time, and then demonstrating the emergence of time and of classical trajectories. Although the Wheeler-DeWitt equation in the form (1.1) is unlikely to be the last word in quantum gravity, it does seem likely that whatever replaces it will still be of a timeless nature. The loop variables programme of Ashtekar and others, for example, certainly preserves this feature [6]. It is therefore of interest to investigate this feature in simple models.

Many attempts to use and make sense of Eq.(1.1) have been made. These attempts focus on simple (minisuperspace) models, in which one has an $N$-dimensional configuration space, $\mathcal{C}$ with coordinates $\mathbf{x}$, and the Hamiltonian operator has the form

$$
H=-\frac{1}{2} \nabla^{2}+V(\mathbf{x})
$$

The signature of the metric is typically hyperbolic so the Wheeler-DeWitt equation is like a Klein-Gordon equation in curved space with a spacetime dependent mass term. Associated 
with it is a Klein-Gordon current

$$
J=i\left(\Psi \nabla \Psi^{*}-\Psi^{*} \nabla \Psi\right)
$$

Like the Klein-Gordon equation, however, this does not produce a positive probability density except in very special cases (namely when there is a Killing vector associated with $H$ ). It also vanishes for real wave functions. One might also consider the Schrödinger inner product,

$$
\left\langle\Psi_{1} \mid \Psi_{2}\right\rangle=\int_{\mathcal{C}} d^{N} \mathbf{x} \mu(\mathbf{x}) \Psi_{1}^{*}(\mathbf{x}) \Psi_{2}(\mathbf{x})
$$

where $\mu(\mathbf{x})$ is an appropriate measure, but the norm $\langle\Psi \mid \Psi\rangle$ typically diverges. In practice, most uses of the Wheeler-DeWitt equation rely on something like the "WKB interpretation", in which in the oscillatory regime the wave function is written in the form $\Psi=C e^{i S}$, where $S$ is a solution to the Hamilton-Jacobi equation. It is argued that this wave function corresponds to an ensemble of classical trajectories satisfying the first integral $p=\nabla S$, with $|C|^{2}$ giving a measure on the ensemble. Although probably correct it is somewhat heuristic and can only be used in the oscillatory regime. (See Ref.[7], for example, for a discussion of these issues).

Recent more successful work with the Wheeler-DeWitt or similar equations involves the induced inner product (also known as Rieffel induction or refined algebraic quantization) $[8,9]$. Here, one considers eigenvalues of the Wheeler-DeWitt operator,

$$
H\left|\Psi_{E k}\right\rangle=E\left|\Psi_{E^{\prime} k^{\prime}}\right\rangle
$$

where $k$ is the degeneracy label for each $E$. The spectrum is typically continuous in $E$ in which case the states are normalized via Eq.(1.4) according to

$$
\left\langle\Psi_{E k} \mid \Psi_{E^{\prime} k^{\prime}}\right\rangle=\delta\left(E-E^{\prime}\right) \delta_{k k^{\prime}}
$$


and one can now see why $\langle\Psi \mid \Psi\rangle$ diverges. The induced inner product is then, loosely speaking, to drop the factor $\delta\left(E-E^{\prime}\right)$ as $E$ and $E^{\prime}$ are set to zero. This procedure can be defined rigorously and induces an inner product on the zero energy eigenstates. (This procedure is not of course necessary when the spectrum is discrete).

Related to these issues is the prevalent idea that any operations performed on the wave function in the computation of physically interesting probabilities should commute with $H$ $[10,3,11]$. Mathematically, this is to respect the symmetry of the theory, reparametrization invariance, expressed by the constraint equation (1.1). Physically, it is connected with the fact that the universe is a genuinely closed system, and all realistic measurements are carried out from the inside, so cannot displace the system from a zero energy eigenstate of $H$.

Given these preliminaries, turn now to the questions one would like to ask of the wave function of the system in order to extract useful cosmological predictions from it. We are interested in the notion that the wave function corresponds in some way to a set of trajectories. Let us therefore ask the question,

What is the probability that the system is found in a series of regions in configuration space, $\Delta_{1}, \Delta_{2}, \cdots \Delta_{n}$ ?

Note that the question is stated in such a way that does not involve time. There is no requirement that the system enters one of the regions at a particular "time", or that the regions are entered in a particular order. We cannot ask this because the WheelerDeWitt equation does not know about such an ordering parameter. In the classical case the corresponding situation consists of a statistical ensemble of classical trajectories with the same fixed energy. The trajectories are simply curves in configuration space and it is straightforward to determine the probability that a given curve passes through a given 
region at any stage in its entire history. The question is more involved in quantum theory, since quantum theory is somewhat resistant to the notion of a trajectory (in the nonrelativistic case, it involves specifying positions at different time, which do no commute). It is, nevertheless, important to develop this notion, since the timeless nature of the WheelerDeWitt equation cries out for an interpretation in terms of entire histories of the universe. The aim of this paper is to offer one possible way of giving meaning to the above question in the quantum theory of simple cosmological models.

Intuitively, one would expect that the question can be formulated and answered using a simple toolbox of parts: the quantum state $|\Psi\rangle$ satisfying the constraint, projection operators onto the regions $\Delta_{k}$, or maybe projections onto some class of operators which commute with the constraint $H$. One might also expect to find the Green function associated with the Wheeler-DeWitt equation, which has the form,

$$
G(\mathbf{x}, \mathbf{y})=i \int_{0}^{\infty} d \tau\left\langle\mathbf{x}\left|e^{-i(H-i \epsilon) \tau}\right| \mathbf{y}\right\rangle=\left\langle\mathbf{x}\left|\frac{1}{(H-i \epsilon)}\right| \mathbf{y}\right\rangle
$$

(this is the analogue of the Feynman propagator). It might possibly also involve one of the other types of propagators obtained by integrating $\tau$ over an infinite range in this expression $[12,13,14]$. The question is then exactly how one stitches all these components together to make a plausible probability distribution describing trajectories passing through a series of regions.

The decoherent histories approach offers an approach to answering this question and it does indeed use some of the above elements [15]. It is particularly adapted to this sort of situation since it directly addresses the issue of defining a quantum notion of "trajectory" and this approach is currently being investigated in this context [16] (see also Ref.[1]). Other approaches involving observables - operators commuting with the constraint - have also been considered $[17,18,9,19]$. Most importantly, Kiefer and Zeh [20] and Barbour 
$[21,22,4]$ have devoted much effort to elucidating the emergence of trajectories and of time from the timeless Wheeler-DeWitt equation.

The approach we adopt here stems from Barbour's observation [22] that a substantial insight into the Wheeler-DeWitt equation may be found in Mott's 1929 analysis of alphaparticle tracks in a Wilson cloud chamber [23]. Mott's paper concerned the question of how the alpha-particle's outgoing spherical wave state, $e^{i k R} / R$, could lead to straight line tracks in a cloud chamber. His explanation was to model the cloud chamber as a collection of atoms that may be ionized by the passage of the alpha-particle. They therefore act as measuring devices that measure the alpha-particle's trajectory. The probability that certain atoms are ionized is indeed found to be strongly peaked when the atoms lie along a straight line through the point of origin of the alpha-particle.

Although Mott seems to have had in mind a time-evolving process, he actually solved the time-independent equation

$$
\left(H_{0}+H_{d}+\lambda H_{\text {int }}\right)|\Psi\rangle=E|\Psi\rangle
$$

Here $H_{0}$ is the alpha-particle Hamiltonian, $H_{d}$ is the Hamiltonian for the ionizing atoms, and $H_{\text {int }}$ describes the Coulomb interaction between the alpha-particle and the ionizing atoms (where $\lambda$ is a small coupling constant). Now the interesting point, as Barbour notes, is that Mott derived all the physics from this equation with little reference to time. Mott's calculation is therefore an excellent model for many aspects of the Wheeler-DeWitt equation. Barbour has elucidated this very eloquently, showing how it sheds light on a number of different aspects $[22,21,4,24]$.

Barbour's discussion is largely qualitative. The point of the present paper, by contrast, is to extract quantitative information from the comparison between the Mott calculation and the Wheeler-DeWitt equation. Mott derived the straight line tracks by looking at the 
wave function associated with two atoms being in the ionized state, for the special case of an outgoing wave initial state. But since this is elementary quantum mechanics, it is a simple matter to generalize it to arbitrary initial states and other types of detectors models, and to derive a detailed expression for the probability distribution. Therefore, Mott's calculation points the way towards a general expression for the probability distribution for the system passing through a series of regions in configuration space without reference to time. This is what we will work out in detail in this paper.

We consider a system in an $N$ dimensional configuration space $R^{N}$ with coordinates $\mathbf{x}$ described by a Hamiltonan $H_{0}$, which may be of the form (1.2), but the simplest case we consider is a free particle. It is coupled to a set of detectors via an interaction $H_{\text {int }}$ and the state of the whole system is given by the solution to (1.8). Mott used the electronic degrees of freedom of atoms as detectors. However, the essence of the calculation is maintained with a much simpler detector model. The detector we use consists of a two state system with $H_{d}=0$ and detector states $|0\rangle$ and $|1\rangle$, where,

$$
a|0\rangle=0, \quad a|1\rangle=|0\rangle, \quad a^{\dagger}|0\rangle=|1\rangle, \quad a^{\dagger}|1\rangle=0
$$

We take

$$
H_{i n t}=\sum_{k} f_{k}(\mathbf{x})\left(a_{k}+a_{k}^{\dagger}\right)
$$

Here, $f_{k}(\mathbf{x})$ is spatially localized in the region $\Delta_{k}$. One could, for example, take $f_{k}$ to be a window function which is 1 in $\Delta_{k}$ and 0 outside it, but we will not restrict to this choice. If the detector in $\Delta_{k}$ is "initially" in the ground state $|0\rangle$, it will be displaced into the excited state $|1\rangle$ if the particle's trajectory $\mathbf{x}(t)$ enters $\Delta_{k}$ and stays in the ground state otherwise. Of course, in the timeless context of the Wheeler-DeWitt equation, "initially" has no meaning. Instead, following Mott, the appropriate condition to impose is that the detector is in the ground state in the absence of coupling to the system. 
This detector is far from realistic, not least of all because it can return to its ground state if the particle spends too much time in the detector region. We will discuss its problems and possible improvements. We note however, that similarly simple detector models have been profitably used elsewhere, e.g., in the Coleman-Hepp model [25]. (See also Ref.[26]).

It is perhaps worth noting that the question considered here bears a close resemblance to the arrival time and tunneling time questions in non-relativistic quantum mechanics $[27,28]$. There also, time enters in a non-trivial way, and equivalent classical approaches to the problem are inequivalent at the quantum level. A variety of approaches have been brought to bear on these problems, including, as here, explicit detector models.

In Section 2, we solve the system Eq.(1.8) using the simple two state detector model.

Using the results, we then ask, in Section 3, some simple questions of the detected wave function. Does the detected wave function still obey the Wheeler-DeWitt equation? We find that it does exactly outside the detector region, and that it does approximately (for small $\lambda$ ) inside the detector region. We compute the probabilities for detection and see, as Mott essentially saw, that they are strongly peaked when the detectors lie along a classical trajectory. We also observe that the resulting amplitude for detection bears a very close resemblance to a formula written down by Hartle [29] (without using an explicit detector model) and we discuss the connections with his result. We also discuss various other aspects of the solution in relation to detection and the timelessness of the solutions.

The results of Section 3 indicate that the wave function of the system may, in some sense be regarded as a superposition of states each of which is peaked about an entire classical history. To demonstrate this explicitly, we specialize, in Section 4, to the case of a collection of harmonic oscillators and introduce a new type of coherent state, the "timeless coherent states". These states are eigenstates of the Hamiltonian, and are therefore time- 
independent, but are peaked about classical trajectories. Any eigenstate may be expanded in terms of theses states, and we show that a series of detections along a classical path essentially projects the state down onto a timeless coherent state.

Since the detector is so simple, its dynamics may be solved exactly and this is carried out in Section 5. This calculation confirms that the detector model is only physical realistic in the perturbative regime (when the particle spends only a short time in each detector

region). Although the solution is exact and leaves the boundary conditions general, it turns out that it is not very useful for the Mott solution, since the boundary conditions lead to a rather inelegant solution. On the other hand, it is by no means clear that one is required to take the Mott boundary conditions for the analagous situation in quantum cosmology, and given the freedom to choose different conditions, an elegant alternative solution for the detector amplitude is obtained. It is in fact almost the same as the amplitude Hartle proposed [29].

In Section 6, we briefly describe a more elaborate detector model, in which the detector is a simple harmonic oscillator coupled to the particle with the same coupling (1.10). The solution has a nice path integral representation and suffers fewer shortcomings that the two-state detector. It also clearly illustrate the peak about classical paths.

We summarize and conclude in Section 7.

\section{DETECTION AMPLITUDE FROM THE TWO-STATE DETECTOR}

We now solve the eigenvalue equation

$$
H|\Psi\rangle=E|\Psi\rangle
$$


with $H$ given by Eq.(1.8), and the detector is the simple two state detector described in Section 1 , with $H_{d}=0$. We will for convenience refer to this equation as the WheelerDeWitt equation (and it is convenient to retain a non-zero value of $E$ ). It now reads

$$
(H-E)|\Psi\rangle=\left(H_{0}+\lambda H_{\text {int }}-E\right)|\Psi\rangle=0
$$

We initially use only two detectors, so

$$
H_{\text {int }}=\sum_{k=1}^{2} f_{k}(\mathbf{x})\left(a_{k}+a_{k}^{\dagger}\right)
$$

but the generalization to a arbitrary number of detectors is straightforward. We solve perturbatively by writing

$$
|\Psi\rangle=\left|\Psi^{(0)}\right\rangle+\lambda\left|\Psi^{(1)}\right\rangle+\lambda^{2}\left|\Psi^{(2)}\right\rangle+\cdots
$$

We require that in the absence of coupling to the detectors, the detectors are in the state of no detection, $|0\rangle$. This means that

$$
\left|\Psi^{(0)}\right\rangle=|\psi\rangle|0\rangle|0\rangle
$$

and $|\psi\rangle$ is the state we are trying to measure. Inserting in (2.2) and equating powers of $\lambda$, we get,

$$
\begin{aligned}
& \left(H_{0}-E\right)\left|\Psi^{(0)}\right\rangle=0 \\
& \left(H_{0}-E\right)\left|\Psi^{(1)}\right\rangle=-H_{i n t}\left|\Psi^{(0)}\right\rangle \\
& \left(H_{0}-E\right)\left|\Psi^{(2)}\right\rangle=-H_{i n t}\left|\Psi^{(1)}\right\rangle
\end{aligned}
$$

and similarly to higher orders. The first relation says that $|\psi\rangle$ must obey the unperturbed eigenvalue equation, as expected. Inserting (2.5) into Eq.(2.7), we get

$$
\left(H_{0}-E\right)\left|\Psi^{(1)}\right\rangle=-f_{1}(\mathbf{x})|\psi\rangle|1\rangle|0\rangle-f_{2}(\mathbf{x})|\psi\rangle|0\rangle|1\rangle
$$


This is readily solved by writing

$$
\left|\Psi^{(1)}\right\rangle=\left|\Psi_{00}^{(1)}\right\rangle|0\rangle|0\rangle+\left|\Psi_{10}^{(1)}\right\rangle|1\rangle|0\rangle+\left|\Psi_{01}^{(1)}\right\rangle|0\rangle|1\rangle+\left|\Psi_{11}^{(1)}\right\rangle|1\rangle|1\rangle
$$

and we discover that

$$
\begin{aligned}
& \left(H_{0}-E\right)\left|\Psi_{00}^{(1)}\right\rangle=0 \\
& \left(H_{0}-E\right)\left|\Psi_{10}^{(1)}\right\rangle=-f_{1}(\mathbf{x})|\psi\rangle \\
& \left(H_{0}-E\right)\left|\Psi_{01}^{(1)}\right\rangle=-f_{2}(\mathbf{x})|\psi\rangle \\
& \left(H_{0}-E\right)\left|\Psi_{11}^{(1)}\right\rangle=0
\end{aligned}
$$

Eqs.(2.12), (2.13) may be solved with the assistance of the Green function $G$, defined by Eq.(1.7) (with $H$ replaced by $H_{0}-E$ ). It obeys the equation

$$
\left(H_{0}-E\right) G=1
$$

(For convenience we use an operator notation in which $G$ is the operator with coordinate representation $G(\mathbf{x}, \mathbf{y})=\langle\mathbf{x}|G| \mathbf{y}\rangle$ and the right-hand side of (2.15) would be the deltafunction $\delta(\mathbf{x}, \mathbf{y})$ in the coordinate representation). We then obtain

$$
\begin{aligned}
\left|\Psi_{10}^{(1)}\right\rangle & =-G f_{1}(\mathbf{x})|\psi\rangle+\left|\phi_{1}\right\rangle \\
\left|\Psi_{01}^{(1)}\right\rangle & =-G f_{2}(\mathbf{x})|\psi\rangle+\left|\phi_{2}\right\rangle
\end{aligned}
$$

where $\left|\phi_{1,2}\right\rangle$ are solutions to the homogeneous equation

$$
\left(H_{0}-E\right)\left|\phi_{1,2}\right\rangle=0
$$

To fix some of these solutions more precisely, we need to appeal to boundary conditions. This is a subtle issue and depends very much on the precise context. Mott was concerned with the particular case of an outgoing spherical wave and imposed boundary conditions 
appropriate to this case. This led him to set $\left|\phi_{1,2}\right\rangle$ and $\left|\Psi_{11}^{(1)}\right\rangle$ to zero (since otherwise it represents a stream of incoming particles fired at an already excited detector) [23]. We are not obviously compelled to make the same choice of boundary conditions in quantum cosmology, and we will return to a discussion of this important issue in Section 3(C). But for the moment, we work with the Mott solution.

At first order only one detector is stimulated into the excited state. The system wave function correlated with detector state $|1\rangle$ now is

$$
\left|\psi_{1}\right\rangle=-\lambda G f_{1}|\psi\rangle
$$

and the probability that the detector registers is therefore

$$
p_{1}=\left\langle\psi_{1} \mid \psi_{1}\right\rangle=\lambda^{2}\left\langle\psi\left|f_{1} G^{\dagger} G f_{1}\right| \psi\right\rangle
$$

(When the spectrum of $H_{0}$ is continuous, expressions of this type need to be regularized along the lines of Eq.(1.6), but we will carry out this explicitly only when we need to calculate it in more detail).

To get two detectors to register, we need to go to second order. We now have, from (2.8), and the solution $(2.10)$,

$$
\begin{aligned}
\left(H_{0}-E\right)\left|\Psi^{(2)}\right\rangle & =-\left(f_{1}\left(a_{1}+a_{1}^{\dagger}\right)+f_{2}\left(a_{2}+a_{2}^{\dagger}\right)\right)\left|\Psi^{(1)}\right\rangle \\
& =f_{2} G f_{1}|\psi\rangle|1\rangle|1\rangle+f_{1} G f_{2}|\psi\rangle|1\rangle|1\rangle+\cdots
\end{aligned}
$$

where the omitted terms on the right-hand side are proportional to $|0\rangle|0\rangle,|0\rangle|1\rangle$ and $|1\rangle|0\rangle$, and will not be needed. Again we may solve by expanding as in (2.10). Here we write down only the term required, which describes two detectors being excited

$$
\left|\Psi^{(2)}\right\rangle=\left|\Psi_{11}^{(2)}\right\rangle|1\rangle|1\rangle+\cdots
$$


and it is readily seen that the solution is

$$
\left|\Psi_{11}^{(2)}\right\rangle=\left(G f_{2} G f_{1}+G f_{1} G f_{2}\right)|\psi\rangle
$$

Again, following the spirit of the Mott solution, possible homogeneous solutions are set to zero. We now have the system wave function correlated with two detectors registering: it is

$$
\left|\psi_{2}\right\rangle=\lambda^{2}\left(G f_{2} G f_{1}+G f_{1} G f_{2}\right)|\psi\rangle
$$

and the probability is $\left\langle\psi_{2} \mid \psi_{2}\right\rangle$.

The analysis is readily extended to an arbitrary number of detectors, but it is easy to anticipate the result from (2.24): for $n$ detectors, the amplitude is

$$
\left|\psi_{n}\right\rangle=\lambda^{n}\left(G f_{n} G f_{n-1} \cdots G f_{2} G f_{1}\right)|\psi\rangle+\text { symmetrizations }
$$

where "symmetrizations" means add all possible permutations of $1,2,3, \cdots n$. It is clear that these terms are there to ensure that there is no preference in the order in which each of the detectors registers, reflecting the genuinely timeless nature of the underlying dynamics. Eq.(2.25) is the main result of this Section.

\section{PROPERTIES OF THE SOLUTION}

We have shown that the wave function for the whole system when there are, for example, two detectors, takes the form

$$
|\Psi\rangle=\left|\psi_{0}\right\rangle|0\rangle|0\rangle+\left|\psi_{1}\right\rangle(|1\rangle|0\rangle+|0\rangle|1\rangle)+\left|\psi_{2}\right\rangle|1\rangle|1\rangle
$$

We can now ask various questions of the detected wave function $\left|\psi_{2}\right\rangle$, or more generally, Eq.(2.25). 


\section{3(A). Does the Detected Wave Function obey the Wheeler-DeWitt Equation?}

As stated in the Introduction, a prevalent idea in quantum gravity is that all observables should commute with the total Hamiltonian $[10,3,11]$. Related to this is the notion that "measurements" of the wave function (whatever this may mean in general) should not displace the system from its eigenstate of the Hamiltonian. Given that we have presented here an explicit model of detection, it is perhaps of interest to ask to what extent this idea holds up.

We have, using (2.15), and taking the simple case $n=2$,

$$
\left(H_{0}-E\right)\left|\psi_{2}\right\rangle=\lambda^{2}\left(f_{2} G f_{1}+f_{1} G f_{2}\right)|\psi\rangle
$$

In configuration space, the right-hand side zero, except in the detector regions $\Delta_{1}, \Delta_{2}$, because the functions $f_{1}$ and $f_{2}$ are localized there. In these regions, it is of order $\lambda^{2}$, which we regard as small in comparison to the terms on the left. Hence the measured wave function approximately obeys the Wheeler-DeWitt equation. This is no surprise. In standard quantum mechanics, a physically measured system does not obey the Schrödinger equation but has corrections due to the measuring device. That it obeys the Wheeler-DeWitt equation only approximately is not in conflict with exact reparametrization invariance, since the wave function for the entire system always obeys the Wheeler-DeWitt equation exactly. On the other hand, one wonders whether it might not be possible to devise a detection scheme in which the detected amplitude obeys the Wheeler-DeWitt equation exactly. For example, given the simple toolbox of parts outlined in the Introduction (such as the Green function (1.7)), it would not be unreasonable to guess that the detection amplitude might be of the form (2.25) but with the $G$ given by Eq.(1.7) replaced by the 
one obtained by integrating $\tau$ over an infinite range. This gives a solution to the WheelerDeWitt equation, and as a consequence, the modified detection amplitude would also obey the Wheeler-DeWitt equation exactly.

\section{3(B). Amplitudes for Classical Paths}

Of greater interest is the question of the configurations about which the amplitude (2.25) (or the associated probability) is peaked. The amplitude may be written in an explicit coordinate representation as

$$
\begin{aligned}
\left\langle\mathbf{x}_{f} \mid \psi_{n}\right\rangle=\lambda^{n} & \int d^{N} \mathbf{x}_{n} \cdots d^{N} \mathbf{x}_{2} d^{N} \mathbf{x}_{1} G\left(\mathbf{x}_{f}, \mathbf{x}_{n}\right) f_{n}\left(\mathbf{x}_{n}\right) \\
& \times G\left(\mathbf{x}_{n}, \mathbf{x}_{n-1}\right) f_{n-1}\left(\mathbf{x}_{n-1}\right) \cdots G\left(\mathbf{x}_{2}, \mathbf{x}_{1}\right) f_{1}\left(\mathbf{x}_{1}\right) \psi\left(\mathbf{x}_{1}\right)
\end{aligned}
$$

(plus symmetrizations). It has the form of approximate projections onto the regions $\Delta_{k}$

(exact projections if the $f_{k}$ are window functions) with evolution between regions described by the fixed energy propagator $G\left(\mathbf{x}_{k+1}, \mathbf{x}_{k}\right)$. It is analogous to the amplitude for a history of positions at a sequence of times in non-relativistic quantum mechanics, which is known to be peaked about classical trajectories [30]. But note that here the evolution is at fixed values of the energy, and there is no reference to time.

We can estimate the form of (3.3) using a WKB approximation for the fixed energy propagator [31]. It is given by an expression of the form,

$$
G\left(\mathbf{x}^{\prime \prime}, \mathbf{x}^{\prime}\right)=C\left(\mathbf{x}^{\prime \prime}, \mathbf{x}^{\prime}\right) e^{i S\left(\mathbf{x}^{\prime \prime}, \mathbf{x}^{\prime}\right)}
$$

where $C$ is a slowly varying prefactor and $S\left(\mathbf{x}^{\prime \prime}, \mathbf{x}^{\prime}\right)$ is the fixed energy Hamilton-Jacobi function, i.e., the action of the classical solution from $\mathbf{x}^{\prime}$ to $\mathbf{x}^{\prime \prime}$ with fixed energy $E$ [32]. The initial and final momenta of this classical solution are

$$
\mathbf{p}^{\prime \prime}=\nabla_{\mathbf{x}^{\prime \prime}} S\left(\mathbf{x}^{\prime \prime}, \mathbf{x}^{\prime}\right), \quad \mathbf{p}^{\prime}=-\nabla_{\mathbf{x}^{\prime}} S\left(\mathbf{x}^{\prime \prime}, \mathbf{x}^{\prime}\right)
$$


In terms of (3.4), the amplitude (3.3) may be written,

$$
\begin{aligned}
\left\langle\mathbf{x}_{f} \mid \psi_{n}\right\rangle=\lambda^{n} \int d^{N} \mathbf{x}_{n} & \cdots d^{N} \mathbf{x}_{2} d^{N} \mathbf{x}_{1} \prod_{k=1}^{n} C\left(\mathbf{x}_{k+1}, \mathbf{x}_{k}\right) f_{k}\left(\mathbf{x}_{k}\right) \\
& \times \exp \left(i \sum_{k=1}^{n} S\left(\mathbf{x}_{k+1}, \mathbf{x}_{k}\right)\right) \psi\left(\mathbf{x}_{1}\right)
\end{aligned}
$$

where $\mathbf{x}_{n+1}=\mathbf{x}_{f}$.

Consider the integrals over $\mathbf{x}_{2} \cdots \mathbf{x}_{n}$ with $\mathbf{x}_{1}$ (and $\left.\mathbf{x}_{n+1}\right)$ fixed. Suppose for the moment that the functions $f_{k}$ are absent so the integrals are unrestricted. By the stationary phase approximation, the dominant contribution to the integral comes from the values of $\mathbf{x}_{2} \cdots \mathbf{x}_{n}$ for which the phase is stationary, i.e., for which

$$
\nabla_{\mathbf{x}_{j}} \sum_{k=1}^{n} S\left(\mathbf{x}_{k+1}, \mathbf{x}_{k}\right)=0
$$

for $j=2, \cdots n$. This equation means that

$$
\nabla_{\mathbf{x}_{j}} S\left(\mathbf{x}_{j+1}, \mathbf{x}_{j}\right)+\nabla_{\mathbf{x}_{j}} S\left(\mathbf{x}_{j}, \mathbf{x}_{j-1}\right)=0
$$

Using Eq.(3.5), this implies that the final momentum of the classical path from $\mathbf{x}_{j-1}$ to $\mathbf{x}_{j}$ is equal to the initial momentum of the classical path from $\mathbf{x}_{j}$ to $\mathbf{x}_{j+1}$. It is not difficult to see that this in turn implies that the point $\mathbf{x}_{j}$ must lie on the classical path from $\mathbf{x}_{j-1}$ to $\mathbf{x}_{j+1}$. Hence, the stationary phase points of the whole integral (3.6) lie on the classical path from $\mathbf{x}_{1}$ to $\mathbf{x}_{f}$. The approximate value of the integral is the integrand of (3.6) with the stationary phase point values inserted.

Now consider what happens when the restricting functions $f_{k}$ are present. If the regions $\Delta_{k}$ (about which the $f_{k}$ are concentrated) include the stationary phase points (and if the regions are larger than the fluctuations about these points), then, since the integral takes it dominant contribution from these points, the presence of the $f_{k}$ makes little difference 
and the integral is given once again by its stationary phase value. On the other hand, if one or more of the $f_{k}$ lie far away from the stationary phase points, then, since the integral is prevented from taking a contribution from these points, its value must be much smaller than the stationary phase value. We thus see that the amplitude (3.3) will be largest when the regions $\Delta_{k}$ are chosen to include the stationary phase points of the integral. As we have shown, the stationary phase points lie along the classical path from $\mathbf{x}_{1}$ to $\mathbf{x}_{f}$. It follows that the amplitude (3.2) will be largest when the regions $\Delta_{k}$ are chosen to lie along a classical path.

Mott's argument for straightline paths in an expression analogous to (2.25) relied on the explicit from of the Green function in the free particle case, and on a special initial state [23]. Here we see that the peaking about classical paths can be seen, at least heuristically, from elementary properties of path integrals, for a broad class of Hamiltonians and initial states. Bell has also discussed the Mott calculation at some length [33]. He notes that the first projector $f_{1}$ spatially localizes Mott's initial wave function, but in a realistic ionizing event, the resultant uncertainty in momentum can still be extremely small. As a consequence the angular spread of the wave packet in its subsequent evolution can be extremely small, hence the appearance of a straight line track.

\section{3(C). Comparison with a Result of Hartle}

The result (2.24) is very closely related to a result of Hartle [29]. He considered a simple model quantum cosmology with a Hamiltonian quadratic in the momenta, as here, and asked for the amplitude that the system passes through two regions of configuration space, $\Delta_{1}, \Delta_{2}$. Using some simple arguments about propagators and elementary principles 
of quantum theory, he showed that the amplitude is (in the language of the present paper)

$$
\left(G f_{2} G f_{1}+G f_{1} G f_{2}\right)|\psi\rangle-\left(G^{\dagger} f_{2} G^{\dagger} f_{1}+G^{\dagger} f_{1} G^{\dagger} f_{2}\right)|\psi\rangle
$$

where $\psi$ is a solution to the Wheeler-DeWitt equation and $f_{1}$ and $f_{2}$ are taken to be exact projectors onto the regions $\Delta_{1}, \Delta_{2}$. Other than the factor of $\lambda^{2}$ in (2.24) which is not important, Hartle's result differs from (2.24) by the subtraction of an identical term but with $G$ replaced by $G^{\dagger}$ (which is generally not the same as $G$ ). Hartle argues that this should be there on the grounds that, in an expression like (2.25) with $G$ represented by (1.7), the time parametrization should not have a preferred direction with $\mathbf{y}$ the "initial" point of the parametrization and $\mathbf{x}$ the "final" point, hence we should sum the amplitude over both possible parametrization directions.

To understand why this term can be there in the present calculation, let us first say a little more about the Green function. $G$, as defined by Eq.(1.7), may be written more explicitly as

$$
G(\mathbf{x}, \mathbf{y}, E)=\sum_{n} \frac{u_{n}^{*}(\mathbf{x}) u_{n}(\mathbf{y})}{E-E_{n}+i \epsilon}
$$

where $u_{n}(\mathbf{x})$ are eigenfunctions of $H_{0}$ with eigenvalue $E_{n} . G(\mathbf{x}, \mathbf{y}, E)$ is real when $E$ lies in the discrete part of the spectrum and complex when $E$ lies in the continuous part (see, for example, Ref.[34]). Hence in the free particle case considered by Mott, $G \neq G^{\dagger}$, but for the harmonic oscillator (considered in Section 4) $G=G^{\dagger}$. Quantum cosmological models usually have a spectrum which is at least in part continuous, so we expect $G \neq G^{\dagger}$ in general.

Now recall the comments after Eq.(2.18), where it was noted that we are by no means obliged in quantum cosmology to take the same boundary conditions as Mott. Since $G^{\dagger}$ also satisfies Eq.(2.15), we may use it in place of $G$ to generate solutions to the detector amplitude. Because $G-G^{\dagger}=i \delta\left(H_{0}-E\right)$, it is easily seen that the difference between 
using $G$ and $G^{\dagger}$ is a homogeneous solution in (2.16), (2.17). It is also true that, in higher order perturbations, we may use $G$ or $G^{\dagger}$ or some combination. Therefore, there is a more general class of solutions for the detection amplitude which are sums of terms of the form (2.25) with some of the $G^{\prime}$ s replaced by $G^{\dagger}$ 's (and with a suitable overall normalization). In particular, Hartle's amplitude falls into this enlarged class of solutions, so there is no conflict with his result.

Mott made a particular choice of solution appropriate to the physical situation he as investigating. In the case of relativistic field theory in Minkowski space, one would normally impose some sort of causality requirement to fix the solution more precisely, and so to choose between $G$ and $G^{\dagger}$ (since $G$ is essentially the Feynman propagator). One could require, for example, that the wave function for the whole system is affected by the detector only in the future light cone of the detector region. In quantum cosmology, however, although the metric has hyperbolic signature like Minkowki space, it is by no means clear that one is obliged to impose an analogous requirement, and in fact it is difficult to see exactly how to do this in general since the configuration space is usually not globally hyperbolic. (See, however, Ref.[12]). Instead, one might expect to fix the choice of $G$ or $G^{\dagger}$ by appealing to cosmological boundary conditions. The no-boundary proposal of Hartle and Hawking, for example, picks out a wave function that is real [35]. It is sometimes claimed that this corresponds to a "time-symmetric" wave function [36]. It therefore stays most closely to the timeless nature of the Wheeler-DeWitt equation and in some sense represents a complete abandonment of any fundamental notion of causality. It is now interesting to note that Hartle's detection wave function (3.8) is in fact real if $\psi$ is real. In many ways this therefore seems like the most natural solution to take in the case of the Wheeler-DeWitt equation. We do not, however, in this paper commit to any particular choice of boundary condition. 
In summary, therefore, a more general solution to the detector model is a sum of terms of the form (2.25) involving both $G$ and $G^{\dagger}$, and this more general solution includes Hartle's result (3.14). Note also that the replacement of $G$ by $G^{\dagger}$ does not affect the discussion of the peaking of the amplitude about classical trajectories.

\section{3(D). On Timelessness and Detection}

It is perhaps worth elaborating on a feature of the detector model which appears at first sight to be incompatible with the timelessness of the Wheeler-DeWitt equation. We have coupled the system to a series of detectors via the interaction Hamiltonian (1.8). This Hamiltonian describes a situation in which, along a trajectory $\mathbf{x}(t)$, the detector is in the ground state "before" the trajectory enters the detector region and in an excited state "after" it has passed through the region. Along a classical trajectory $\mathbf{x}(t)$ in which there is a notion of time, and of before and after, this is undeniably correct. (The parameter $t$ simply labels the points along the curve - it may be take to be, for example, the distance along the curve from some reference point.) But how are we to understand how the detector works in the genuinely timeless world described by the Wheeler-DeWitt equation? There is no before and after and there is no preferred direction of time.

The above results effectively imply that each solution to the Wheeler-DeWitt equation may be regarded as a superposition of states each of which is concentrated along an entire classical trajectory in configuration space (and we will see this in more detail in Section 4). What then seems to be happening in the present model including the detector, is that each trajectory carries a label indicating whether or not it passes through the detector region at any stage along its entire length. In the (perhaps restrictive) language of time, at each point along the trajectory, the label allows the trajectory to "know" whether it 
passed through the detector region in the past, or will pass through it in the future. But it is only on adopting this temporal language that the situation seems paradoxical. The paradox vanishes when ones speaks the vocabulary of entire trajectories in configuration space, and one can see this in the solution (3.1). The wave function of the entire system is written as a correlated state in which the state correlated with the detectors in the $|1\rangle$ state is the state (2.25): the detected state is indeed concentrated on trajectories that pass through $\Delta_{1}, \cdots \Delta_{n}$.

The issues discussed in this Section may be of relevance to the perennial debate on the question of time asymmetry in quantum cosmology $[37,20]$.

\section{COHERENT STATES FOR TIMELESS DYNAMICS}

Since the peaking about classical paths is the most important property of the amplitude (2.25), it is worth exploring it in more detail for the special case of a collection of harmonic oscillators, where it is possible to show very clearly how the solutions to the WheelerDeWitt equation correspond to superpositions of states peaked about classical paths. We will introduce a class of coherent states appropriate to the timeless theories considered here and which are natural analogues of the standard coherent states of the harmonic oscillator.

The Hamiltonian for a set of $N$ identical harmonic oscillators is

$$
H_{0}=\frac{1}{2}\left(\mathbf{p}^{2}+\mathbf{x}^{2}\right)
$$

In this case the spectrum of $H_{0}$ is discrete and we have

$$
\delta\left(H_{0}-E\right)=\int_{0}^{2 \pi} \frac{d t}{2 \pi} e^{-i\left(H_{0}-E\right) t}
$$


Since $\delta\left(H_{0}-E\right)$ is now in fact a true projection operator we may write $\delta\left(H_{0}-E\right)^{2}=$ $\delta\left(H_{0}-E\right)$ without having to worry about regularization through the induced inner product, as in the continuous case. (In this expression $E$ is allowed to take only the discrete values corresponding to the spectrum of $\left.H_{0}\right)$. The Green function $G$ is given as before by (1.7) with $H$ replaced by $H_{0}-E$. For a one-dimensional oscillator, Eq.(4.2) is equivalent to

$$
\delta\left(H_{0}-E\right)=|E\rangle\langle E|
$$

where $|E\rangle$ is the energy eigenstate. In more than one dimension the energy eigenstates are degenerate, so Eq.(4.2) has the form

$$
\delta\left(H_{0}-E\right)=\sum_{d}|E, d\rangle\langle E, d|
$$

where $|E, d\rangle$ are the energy eigenstates with degeneracy label $d$.

The standard coherent states (see Ref.[38], for example) are denoted $|\mathbf{p}, \mathbf{x}\rangle$ and they have the important property that they are preserved in form under unitary evolution,

$$
e^{-i H_{0} t}|\mathbf{p}, \mathbf{x}\rangle=\left|\mathbf{p}_{t}, \mathbf{x}_{t}\right\rangle
$$

where $\mathbf{p}_{t}, \mathbf{x}_{t}$ are the classical solutions matching $\mathbf{p}, \mathbf{x}$ at $t=0$, hence they are strongly peaked about the classical path. We are interested in finding a set of states which are analogues of these states for the timeless case. That is, they should be eigenstates of $H_{0}$, and should be peaked about the classical paths of given fixed energy in phase space. It is not difficult to see that a set of states doing the job are,

$$
\begin{aligned}
\left|\phi_{\mathbf{p x}}\right\rangle & =\delta\left(H_{0}-E\right)|\mathbf{p}, \mathbf{x}\rangle \\
& =\int_{0}^{2 \pi} \frac{d t}{2 \pi} e^{-i\left(H_{0}-E\right) t}|\mathbf{p}, \mathbf{x}\rangle \\
& =\int_{0}^{2 \pi} \frac{d t}{2 \pi} e^{i E t}\left|\mathbf{p}_{t}, \mathbf{x}_{t}\right\rangle
\end{aligned}
$$


These states are not in fact normalized to unity but we shall see that it is useful to work with them as they are. Since the states $\left|\mathbf{p}_{t}, \mathbf{x}_{t}\right\rangle$ are concentrated at a phase space point for each $t$, clearly integrating $t$ over a whole period produces a state which is concentrated along the entire classical trajectory. Each state is labeled by a fiducial phase space point $\mathbf{p}, \mathbf{x}$ which determines the classical trajectory the state is peaked about. Under evolution of the fiducial point $\mathbf{p}, \mathbf{x}$ to another point, $\mathbf{p}_{s}, \mathbf{x}_{s}$, say, along the same classical trajectory, the state changes by a phase,

$$
\left|\phi_{\mathbf{p x}}\right\rangle \rightarrow\left|\phi_{\mathbf{p}_{s} \mathbf{x}_{s}}\right\rangle=e^{i E s}\left|\phi_{\mathbf{p x}}\right\rangle
$$

as may be seen from (4.5), (4.6). We will refer to these states as timeless coherent states. Their properties are in fact very similar to the usual coherent states.

Note that the coherent states $|\mathbf{p}, \mathbf{x}\rangle$ are in fact already approximate eigenstates of $H_{0}$, with eigenvalue $\frac{1}{2}\left(\mathbf{p}^{2}+\mathbf{x}^{2}\right)$, as long as $|\mathbf{p}|,|\mathbf{x}|$ are much larger than the coherent state quantum fluctuations. Given $E$, it therefore seems reasonable to choose the values $\mathbf{p}, \mathbf{x}$ in the fiducial coherent state so that $E=\frac{1}{2}\left(\mathbf{p}^{2}+\mathbf{x}^{2}\right)$, when constructing the timeless states (4.6).

Two timeless coherent states of different energy are exactly orthogonal. The more interesting case is that in which they have the same energy, and then they are approximately orthogonal if they correspond to sufficiently distinct classical solutions. This is because we have

$$
\begin{aligned}
\left\langle\phi_{\mathbf{p}^{\prime} \mathbf{x}^{\prime}} \mid \phi_{\mathbf{p x}}\right\rangle & =\left\langle\mathbf{p}^{\prime}, \mathbf{x}^{\prime}\left|\delta\left(H_{0}-E\right)\right| \mathbf{p}, \mathbf{x}\right\rangle \\
& =\int_{0}^{2 \pi} \frac{d t}{2 \pi} e^{i E t}\left\langle\mathbf{p}^{\prime}, \mathbf{x}^{\prime} \mid \mathbf{p}_{t}, \mathbf{x}_{t}\right\rangle
\end{aligned}
$$

From the properties of the standard coherent states we know that

$$
\left|\left\langle\mathbf{p}^{\prime}, \mathbf{x}^{\prime} \mid \mathbf{p}_{t}, \mathbf{x}_{t}\right\rangle\right| \leq 1
$$


with equality if and only if $\mathbf{p}^{\prime}=\mathbf{p}_{t}$ and $\mathbf{x}^{\prime}=\mathbf{x}_{t}$. Moreover, the overlap of two coherent states is exponentially small if they are centered around phase space points that are sufficiently far apart. It follows that if $\mathbf{p}^{\prime}, \mathbf{x}^{\prime}$ does not lie on, or close to, the trajectory $\mathbf{p}_{t}, \mathbf{x}_{t}$, the overlap $\left\langle\mathbf{p}^{\prime}, \mathbf{x}^{\prime} \mid \mathbf{p}_{t}, \mathbf{x}_{t}\right\rangle$ will always exponentially small for all $t$. The integral over $t$ in (4.8) will then give a result that is much smaller than the case in which $\mathbf{p}^{\prime}, \mathbf{x}^{\prime}$ does lie on, or close to, the trajectory $\mathbf{p}_{t}, \mathbf{x}_{t}$ (because in the latter case the overlap $\left\langle\mathbf{p}^{\prime}, \mathbf{x}^{\prime} \mid \mathbf{p}_{t}, \mathbf{x}_{t}\right\rangle$ becomes close to unity for some value of $t$ ). The timeless coherent states are therefore approximately orthogonal for sufficiently distinct classical trajectories.

The standard completeness relation for the coherent states is

$$
\int \frac{d^{N} \mathbf{p} d^{N} \mathbf{x}}{(2 \pi)^{N}}|\mathbf{p x}\rangle\langle\mathbf{p x}|=1
$$

Multiplying both sides by $\delta\left(H_{0}-E\right)$ from the left and right, and using (4.6), we get

$$
\int \frac{d^{N} \mathbf{p} d^{N} \mathbf{x}}{(2 \pi)^{N}}\left|\phi_{\mathbf{p} \mathbf{x}}\right\rangle\left\langle\phi_{\mathbf{p x}}\right|=\delta\left(H_{0}-E\right)
$$

Since $\delta\left(H_{0}-E\right)|\psi\rangle=|\psi\rangle$ on any solution to the eigenvalue equation $\left(H_{0}-E\right)|\psi\rangle=0$, this is as good as a completeness relation on the set of solutions to the eigenvalue equation (which is all we are interested in). We may therefore write any solution $|\psi\rangle$ as a superposition of timeless coherent states,

$$
|\psi\rangle=\int \frac{d^{N} \mathbf{p} d^{N} \mathbf{x}}{(2 \pi)^{N}}\left|\phi_{\mathbf{p} \mathbf{x}}\right\rangle\left\langle\phi_{\mathbf{p} \mathbf{x}} \mid \psi\right\rangle
$$

It is then tempting to interpret $\left\langle\phi_{\mathbf{p x}} \mid \psi\right\rangle$ as the amplitude that a system in the state $|\psi\rangle$ will be found on the classical trajectory corresponding to the timeless coherent state $\left|\phi_{\mathbf{p x}}\right\rangle$. Again using the fact that $\delta\left(H_{0}-E\right)|\psi\rangle=|\psi\rangle$ it is easy to see that this is in fact the same as $\langle\mathbf{p}, \mathbf{x} \mid \psi\rangle$, which is the amplitude for finding the system at the phase space point $\mathbf{p}, \mathbf{x}$ labeling the trajectory. The probability is then simply $|\langle\mathbf{p}, \mathbf{x} \mid \psi\rangle|^{2}$. This is a simple and 
intuitively appealing result: the classical trajectory is completely fixed by its initial values $\mathbf{x}, \mathbf{p}$, hence we expect that the probability for being found on a certain classical trajectory is the same as the probability for being found at the initial phase space point that labels it.

This interpretation is put forward with a small note of caution, however, since the sum over $\mathbf{p}, \mathbf{x}$ in Eq.(4.12) is not only over states which are only approximately orthogonal (as with the usual coherent states), but, because of the property (4.7), includes some redundancy in the summation. In particular, $\left|\phi_{\mathbf{p x}}\right\rangle\left\langle\phi_{\mathbf{p x}}\right|$ is invariant along the classical phase space trajectory of its fiducial point. Since this only produces some sort of constant factor, it may not make any difference, and indeed, the above interpretation appears to produce sensible results. Still, it would be desirable to include, if possible, some sort of "gauge fixing" which factors out this redundancy. This will be explored elsewhere.

Given all of this background, we may now reconsider the detector amplitude (2.25). We expand the initial state $|\psi\rangle$ in the timeless coherent states, as in Eq.(4.12). When $f_{1}$ operates on the state, it has the effect of restricting the sum over timeless coherent states to only those that pass through the region $\Delta_{1}$, but otherwise leaves the timeless coherent states preserved in form (as long as the region $\Delta_{1}$ is larger than the width of the coherent states). Next, to consider the operation of $G$, we write it as

$$
G=i \int_{0}^{\infty} d \tau e^{-i \tau(H-i \epsilon)}
$$

where $H=H_{0}-E$. When the timeless coherent states are operated on with the exponential factor in (4.13) it has essentially no effect, since $H\left|\phi_{\mathbf{p x}}\right\rangle=0$. (The time integration leads to a constant factor, which may, however, need regulating along the lines of (1.6)). Operating with $f_{2}$ further restricts the sum to those states that pass through both $\Delta_{1}$ and $\Delta_{2}$, and then subsequent operation $G$ again does essentially nothing. And likewise to the end of the 
chain. We therefore find that the detector amplitude (2.25) consists of a superposition of only those timeless coherent states that pass through all the detector regions $\Delta_{1}, \Delta_{2} \cdots \Delta_{n}$. This could be quite a large sum of states if the regions $\Delta_{k}$ are large. However, it will consist of essentially just one timeless coherent state if the detector regions lie along a classical trajectory and if their size is just bigger than the spatial width of the wave packet. The detection amplitude along this classical trajectory is then equal to $\left\langle\phi_{\mathbf{p x}} \mid \psi\right\rangle$, in agreement with the analysis based on Eq.(4.12).

Rovelli has written down a coherent state of the type considered here [17] in the context of a very similar model, although its properties were not explored and exploited as they are here. Klauder, in his approach to the quantization of constrained systems using coherent states, considered the projection of the standard coherent states onto the contraint subspace, hence in essence wrote down states of the form (4.6) [39]. He did not, however, consider their use as an interpretational tool. Wave packet solutions to the Wheeler-DeWitt equation which aproximately track the classical trajectories for more interesting cosmological models have been considered in Refs.[40,41].

\section{EXACT SOLUTION TO THE DETECTOR DYNAMICS}

In this Section we discuss an alternative method of solution of the eigenvalue equation (2.2). As we shall see, it does not in fact give a very elegant representation of the Mott solution, which is why it was not used above. If, however, one is permitted to use different boundary conditions, as may be reasonable in quantum cosmology, then it provides an alternative possible solution to the detector dynamics.

We consider first the case of a single detector. The key observation is that solution to 
the eigenvalue equation (2.2) may be generated using either of the expressions,

$$
\begin{aligned}
|\Psi\rangle & =\delta(H-E)|\phi\rangle \\
& =\frac{1}{2 \pi} \int_{-\infty}^{\infty} d \tau e^{-i \tau\left(H_{0}+\lambda H_{i n t}-E\right)}|\phi\rangle
\end{aligned}
$$

Here $|\phi\rangle$ is an arbitrary fiducial state in the joint system-detector Hilbert space. It is ambiguous up to the addition of a term of the form $(H-E)\left|\phi^{\prime}\right\rangle$. For the moment we keep $|\phi\rangle$ general and take

$$
|\phi\rangle=\left|\chi_{0}\right\rangle|0\rangle+\left|\chi_{1}\right\rangle|1\rangle
$$

To evaluate (5.1) or (5.2) we introduce the eigenstates of $a+a^{\dagger}$, which are

$$
| \pm\rangle=\frac{1}{\sqrt{2}}(|0\rangle \pm|1\rangle)
$$

The expression (5.1) is then readily evaluated with the result

$$
|\Psi\rangle=\left|\psi_{n d}\right\rangle|0\rangle+\left|\psi_{d}\right\rangle|1\rangle
$$

where,

$$
\begin{aligned}
\left|\psi_{n d}\right\rangle & =\frac{1}{2}\left(\delta\left(h+\lambda f_{1}\right)+\delta\left(h-\lambda f_{1}\right)\right)\left|\chi_{0}\right\rangle+\frac{1}{2}\left(\delta\left(h+\lambda f_{1}\right)-\delta\left(h-\lambda f_{1}\right)\right)\left|\chi_{1}\right\rangle \\
\left|\psi_{d}\right\rangle & =\frac{1}{2}\left(\delta\left(h+\lambda f_{1}\right)-\delta\left(h-\lambda f_{1}\right)\right)\left|\chi_{0}\right\rangle+\frac{1}{2}\left(\delta\left(h+\lambda f_{1}\right)+\delta\left(h-\lambda f_{1}\right)\right)\left|\chi_{1}\right\rangle
\end{aligned}
$$

(recalling that $h=H-E$ ). These expressions are perhaps more easily appreciated using a path integral respresentation:

$$
\begin{aligned}
\psi_{n d}\left(\mathbf{x}_{f}\right)= & \int_{-\infty}^{\infty} d \tau e^{i \tau E} \int \mathcal{D} \mathbf{x}(t) \exp (i S[\mathbf{x}(t)]) \\
& \times\left[\cos \left(\lambda \int_{0}^{\tau} d t f_{1}(\mathbf{x}(t))\right) \chi_{0}\left(\mathbf{x}_{0}\right)+i \sin \left(\lambda \int_{0}^{\tau} d t f_{1}(\mathbf{x}(t))\right) \chi_{1}\left(\mathbf{x}_{0}\right)\right] \\
\psi_{d}\left(\mathbf{x}_{f}\right)= & \int_{-\infty}^{\infty} d \tau e^{i \tau E} \int \mathcal{D} \mathbf{x}(t) \exp (i S[\mathbf{x}(t)]) \\
& \times\left[i \sin \left(\lambda \int_{0}^{\tau} d t f_{1}(\mathbf{x}(t))\right) \chi_{0}\left(\mathbf{x}_{0}\right)+\cos \left(\lambda \int_{0}^{\tau} d t f_{1}(\mathbf{x}(t))\right) \chi_{1}\left(\mathbf{x}_{0}\right)\right]
\end{aligned}
$$


Eqs.(5.8), (5.9) represent the exact solution to Eq.(2.2) with one detector in place.

Turn now to the question of the fiducial state. The condition (2.5), suggests that we should take

$$
|\phi\rangle=\left|\chi_{0}\right\rangle|0\rangle
$$

where $|\psi\rangle=\delta\left(H_{0}-E\right)\left|\chi_{0}\right\rangle$, and hence that $\left|\chi_{1}\right\rangle=0$. In fact, in the induced inner product scheme, we may take $\left|\chi_{0}\right\rangle=|\psi\rangle$, since effectively $\delta\left(H_{0}-E\right)^{2}=\delta\left(H_{0}-E\right)$. This therefore yields a path integral expression for the amplitude for detection and has the property that the factoring condition (2.5) is satisfied when $\lambda=0$. So let us first explore the properties of (5.8), (5.9) with $\chi_{1}=0$.

The nature of the sums over paths $\mathbf{x}(t)$ in $\operatorname{Eq}(5.8)$ and (5.9) is governed by the quantity

$$
\tau_{d}=\int_{0}^{\tau} d t f_{1}(\mathbf{x}(t))
$$

appearing in the sine and cosine factors. With $f_{1}$ normalized to be dimensionless, this quantity has the dimensions of time, and is essentially the time spent by the path $\mathbf{x}(t)$ in the region $\Delta_{1}$ around the detector. (This is not of course a physically measurable time. The paths $\mathbf{x}(t)$ in the path integral have a well-defined notion of time, but after summing over the total time duration of each path $\tau$ the final result is time-independent). Now, in the amplitude for no detection, (5.8), the factor $\cos \left(\lambda \tau_{d}\right)$ is 1 for $\tau_{d}=0$, and decreasing for $\tau_{d}$ increasing from zero. In the path integral, it therefore has the effect of suppressing paths that pass close to the detector, and favours paths that stay away from it. Similarly, in the detection amplitude, $(5.9)$, the factor $\sin \left(\lambda \tau_{d}\right)$ is zero for paths that spend no time near the detector, and non-zero for paths that enter the detector region. It therefore enhances the amplitude for paths entering the detector region. The sine and cosine factors therefore, in a very crude way, enforce restrictions on the paths corresponding to entering or not entering the detector region, as one would expect. We can also see, however, that these factors only 
do their job well if $\lambda \tau_{d}$ is somewhat smaller than 1 , indicating that the detector model is only physically sensible in the perturbative regime about $\lambda=0$, as expected.

It is now important to check the agreement between the exact result above and the perturbative result of Section 2. It is not difficult to show that the exact solution (5.9) with $\chi_{1}=0$ does not in fact agree with the perturbative solution (2.19). It differs by the presence of homogeneous solutions in the small $\lambda$ limit of (5.9). We will not go into details but it may be shown using (5.7) and the identity,

$$
\delta(h+\lambda f)=\delta(h)+i \lambda\left(G f G-G^{\dagger} f G^{\dagger}\right)+O\left(\lambda^{2}\right)
$$

(which is proved using the exponential representation (5.2)). Recall that in the perturbative solution the homogeneous solutions were removed at each order in perturbation theory essentially by inspection. Since no corresponding condition has been imposed here, it is not surprising that these spurious solutions crop up. We can, however, get agreement if we choose $\left|\chi_{1}\right\rangle=i \lambda f G^{\dagger}\left|\chi_{0}\right\rangle$ in Eq.(5.9) (again proved using (5.12), although there is no obvious independent reason for making this choice. Furthermore, the presence of the Green function $G^{\dagger}$ in the fiducial state rather destroys the elegance of the path integral representation compared to the case $\chi_{1}=0$.

On the other hand, although the Mott solution is not readily obtained, the removal of the homogeneous solutions in the perturbative solution is a subtle matter of boundary conditions, above and beyond the basic factoring condition (5.10). As discussed in Section 3, in the case of quantum cosmology one is not obviously obliged to take the same conditions, and indeed one can argue that, beyond (5.10), the boundary conditions are up for grabs. Indeed, one could effectively choose boundary conditions by proposing that the solution is given by the formula (5.1) with the factoring condition (5.10). As one can see from (5.12), this proposal again produces solutions with a time symmetric flavour to them 
(i.e., with an equal number of $G^{\prime}$ s and $G^{\dagger}$ 's in the solution), a feature which persists to the case of more than one detector. Not surprisingly, this choice does in fact produce, in essence, the Hartle amplitude (3.8).

We have, therefore, in this Section produced another candidate expression for the detection amplitude, which is arguably the more appropriate one for quantum cosmology. Furthermore, the means of generating it, Eqs.(5.1), (5.2), are readily generalizable to more complicated situations.

\section{AN IMPROVED DETECTOR MODEL}

We now briefly consider a more elaborate detector model that consists of a harmonic oscillator instead of the simple two state system. So we take, in the case of a single detector,

$$
H_{d}=\omega a^{\dagger} a, \quad H_{i n t}=f(\mathbf{x})\left(a+a^{\dagger}\right)
$$

The energy eigenstate of the total Hamiltonian is again calculated using (5.2), with the factored fiducial state (5.10), where $|0\rangle$ is the harmonic oscillator ground state. In terms of a path integral, denoting the harmonic oscillator coordinates by $q$,

$$
\begin{aligned}
\Psi\left(\mathbf{x}_{f}, q_{f}\right)= & \int_{-\infty}^{\infty} d \tau e^{i E \tau} \int \mathcal{D} \mathbf{x}(t) \mathcal{D} q(t) \\
& \times \exp \left(i S_{0}[\mathbf{x}(t)]+i S_{d}[q(t)]+i \lambda S_{i n t}[q(t), \mathbf{x}(t)]\right) \quad \chi_{0}\left(\mathbf{x}_{0}\right) u_{0}\left(q_{0}\right)
\end{aligned}
$$

where $u_{0}(q)=\langle q \mid 0\rangle$. The integral over $q$ is conveniently rewritten as

$$
\begin{array}{r}
\int \mathcal{D} q(t) \exp \left(i S_{d}[q(t)]+i \lambda S_{i n t}[q(t), \mathbf{x}(t)]\right) u_{0}\left(q_{0}\right) \\
=\left\langle q_{f}\left|T \exp \left(-i \tau\left(H_{d}+\lambda H_{i n t}\right)\right)\right| 0\right\rangle
\end{array}
$$


where $T$ denotes time ordering. The right-hand side of (6.3) is just the unitary evolution of the vacuum state for a driven harmonic oscillator. Using the properties of coherent states [38], (6.3) is equal to $\left\langle q_{f} \mid z(\tau)\right\rangle$, where $|z\rangle$ is a standard coherent state and

$$
z(\tau)=-i \lambda \int_{0}^{\tau} d t e^{i \omega(\tau-t)} f(\mathbf{x}(t))
$$

To find the amplitudes for detection and no detection we expand the total state (6.2) in terms of the eigenstates of the harmonic oscillator. Since all states other than the ground state correspond to detection, there is no single amplitude corresponding to detection (although there is a probability). It is therefore easier to look at the amplitude for no detection, which is obtained by overlapping $(6.2)$ with the ground state $u_{0}\left(q_{f}\right)$, yielding,

$$
\psi_{n d}\left(\mathbf{x}_{f}\right)=\int_{-\infty}^{\infty} d \tau e^{i E \tau} \int \mathcal{D} \mathbf{x}(t)\langle 0 \mid z(\tau)\rangle \exp \left(i S_{0}[\mathbf{x}(t)]\right) \chi_{0}\left(\mathbf{x}_{0}\right)
$$

From the properties of coherent states [38], we have

$$
\langle 0 \mid z(\tau)\rangle=\exp \left(-\frac{1}{2}|z(\tau)|^{2}\right)
$$

The probability for no detection is $\left\langle\psi_{n d} \mid \psi_{n d}\right\rangle$ and the probability for detection is simply $1-\left\langle\psi_{n d} \mid \psi_{n d}\right\rangle$

The result (6.5) clearly has the desired properties. For paths $\mathbf{x}(t)$ which never enter the detection region, $z(\tau)=0$ and the path integral is unaffected. Paths that enter the region, on the other hand, generally have $z(\tau) \neq 0$, and they are exponentially suppressed. This is therefore a much improved detector model in comparison to Eq.(5.8). Its validity is not restricted to the perturbative regime. The generalization to many detectors is trivial and essentially the same result concerning peaking about classical trajectories is the obtained.

Of course, this detector is still not fully satisfactory because it can happen that $z(\tau)=0$ even for paths that enter the region, because of the oscillatory nature of $z(\tau)$, hence we 
again encounter the issue of detector recurrences. Again this will be avoided if the time the trajectory spends in the detector region is short (less than $\omega^{-1}$ ).

A more challenging detector improvement avoiding the recurrence problem would be to construct one that, were it used in standard unitary quantum mechanics, would be irreversible, i.e., involves an essentially infinite number of degrees of freedom. Such a

detector was introduced in the related context of measuring arrival times in Ref.[42] and it would be interesting to incorporate it into the situation considered here.

\section{SUMMARY}

The aim of this paper was to give substance to the appealing intuitive notion that solutions to the Wheeler-DeWitt equation (1.1) correspond to entire histories of the universe with time emerging as a parameter along each trajectory. The concrete technical results

- the detection amplitude and the introduction of a set of timeless coherent states - are compatible with this notion. There are, however, many subtle aspects to this notion [4], and we do not claim to have an exhaustive demonstration of the emergence of trajectories from the Wheeler-DeWitt equation.

\section{ACKNOWLEDGEMENTS}

I am very grateful to Julian Barbour, Jim Hartle, John Klauder and Dieter Zeh for their comments on the first draft of this paper. 


\section{REFERENCES}

1. J.B.Hartle, in Proceedings of the 1992 Les Houches School, Gravity and its Quantizations, edited by B.Julia and J.Zinn-Justin (Elsevier Science B.V. 1995)

2. C.J.Isham, gr-qc/9210011.

3. K.Kuchar, in Conceptual Problems of Quantum Gravity, edited by A.Ashtekar and J.Stachel (Boston, Birkhauser, 1991); and in Proceedings of the 4 th Canadian Conference on General Relativty and Relativistic Astrophysics, edited by G.Kunstatter, D.E.Vincent and J.G.Williams (World Scientific, New Jersey, 1992). See also the e-print gr-qc/9304012, Canonical quantum gravity.

4. J.Barbour, The End of Time: The Next Revolution in our Understanding of the Universe (Weidenfeld and Nicholson, 1999).

5. J.Butterfield and C.J.Isham, gr-qc/9901024.

6. For a nice review see, C.Rovelli, gr-qc/9710008, Loop quantum gravity.

7. J.J.Halliwell, in, Proceedings of the 13th International Conference on General Relativity and Gravitation, edited by R.J.Gleiser, C.N.Kozameh, O.M.Moreschi (IOP Publishers, Bristol,1992). (Also available as the e-print gr-qc/9208001).

8. A.Ashtekar, J.Lewandowski, D.Marolf, J.Mourao and T.Thiemann, J.Math.Phys. 36, 6456 (1995); A.Higuchi, Class. Quant. Grav. 8, 1983 (1991). D.Giulini and D.Marolf, Class. Quant. Grav. 16, 2489 (1999); Class. Quant. Grav. 16, 2479 (1999). F.Embacher, Hadronic J. 21, 337 (1998); N.Landsmann, J.Geom.Phys. 15, 285 (1995).

9. D.Marolf, Class. Quant. Grav. 12, 1199 (1995). 
10. B.DeWitt, in Gravitation: An Introduction to Current Research, edited by L.Witten (John WIley and Sons, New York, 1962).

11. C.Rovelli, Class. Quant. Grav. 8, 297 (1991); 8, 317 (1991).

12. C.Teitelboim, Phys. Rev. D25, 3159 (1983); 28, 297 (1983); 28, 310 (1983).

13. J.J.Halliwell, Phys. Rev. D38, 2468 (1988).

14. J.J.Halliwell and M.E.Ortiz, Phys.Rev. D48, 748 (1993).

15. M.Gell-Mann and J.B.Hartle, in Complexity, Entropy and the Physics of Information, SFI Studies in the Sciences of Complexity, Vol. VIII, W. Zurek (ed.) (Addison Wesley, Reading, 1990); and in Proceedings of the Third International Symposium on the Foundations of Quantum Mechanics in the Light of New Technology, S. Kobayashi, H. Ezawa, Y. Murayama and S. Nomura (eds.) (Physical Society of Japan, Tokyo, 1990); Phys.Rev. D47, 3345 (1993); R.B.Griffiths, J.Stat.Phys. 36, 219 (1984); Phys.Rev.Lett. 70, 2201 (1993); Am.J.Phys. 55, 11 (1987); R.Omnès, J.Stat.Phys. 53, 893 (1988); 53, 933 (1988); 53, 957 (1988); 57, 357 (1989); 62, 841 (1991); Ann.Phys. 201, 354 (1990); Rev.Mod.Phys. 64, 339 (1992); J.B.Hartle, in Quantum Cosmology and Baby Universes, S. Coleman, J. Hartle, T. Piran and S. Weinberg (eds.) (World Scientific, Singapore, 1991); J.J.Halliwell, in Fundamental Problems in Quantum Theory, edited by D.Greenberger and A.Zeilinger, Annals of the New York Academy of Sciences, Vol 775, 726 (1994). For further developments in the decoherent histories approach, particularly adpated to the problem of spacetime coarse grainings, see C. Isham, J. Math. Phys. 23, 2157 (1994); C. Isham and N. Linden, J. Math. Phys. 35, 5452 (1994); 36, 5392 (1995).

16. J.J.Halliwell and J.Thorwart, in preparation. See also the related works, J.Whelan, 
Phys. Rev. D50, 6344 (1994); J.B.Hartle and D.Marolf, Phys. Rev. D56, 6247 (1997).

17. C.Rovelli, Phys. Rev. 42, 2638 (1990).

18. C.Rovelli, Phys. Rev. 43, 442 (1991).

19. D.Marolf, Phys. Rev. D53, 6979(1996); Class. Quant. Grav. 12, 2469 (1995); Class. Quant. Grav. 12, 1441 (1995).

20. H.D.Zeh, The Physical Basis of the Direction of Time, third edition (Springer-Verlag, 1999) (and the associated webpage www.time-direction.de); Phys.Lett. A116, 9 (1986); A126, 311 (1988); C.Kiefer and H.Zeh, Phys.Rev. D51, 4145 (1995).

21. J.Barbour, Class. Quant. Grav. 11, 2853 (1994).

22. J.Barbour, Class. Quant. Grav. 11, 2875 (1994).

23. N.F.Mott, Proc.Roy.Soc A124, 375 (1929), reprinted in Quantum Theory and Measurement, edited by J.Wheeler and W.Zurek (Princeton University Press, Princeton, New Jersey, 1983).

24. See also, A.A.Broyles, Phys. Rev. A48, 1055 (1993), and M.Castagnino and R.Laura, gr-qc/0006012, for further discussions of the Mott calculation.

25. K.Hepp, Helv.Phys.Acta 45, 237 (1972).

26. This detector model was used in a simple non-relativistic context by J.J.Halliwell, Phys. Rev. D60, 105031 (1999). Some subsequent developments of the ColemanHepp model are H.Nakazato and S.Pascazio, Phys. Rev. Lett. 70, 1 (1993); Phys. Rev. A48, 1066 (1993); R.Blasi, S.Pascazio, S.Takagi, Phys. Rev. A250, 230 (1998).

27. Y.Aharanov and D.Bohm, Phys. Rev. 122, 1649 (1961); Y.Aharanov, J.Oppenheim, 
S.Popescu, B.Reznik and W.Unruh, quant-ph/9709031 (1997); G.R.Allcock, Ann. Phys. (N.Y.) 53, 253 (1969); 53, 286 (1969); 53, 311 (1969); Ph.Blanchard and A.Jadczyk, Helv.Phys.Acta. 69, 613 (1996); I.Bloch and D.A.Burba, Phys. Rev. 10, 3206 (1974); V.Delgado, preprint quant-ph/9709037 (1997); R.Giannitrapani, preprint quant-ph/9611015 (1998); N.Grot, C.Rovelli and R.S.Tate, Phys. Rev. A54, 46 (1996); E.Gurjoy and D.Coon, Superlattices and Microsctructures 5, 305 (1989); A.S.Holevo, Probabilistic and Statistical Aspects of Quantum Theory (North Holland, Amsterdam, 1982), pages 130-197; A.Jadcyk, Prog.Theor.Phys. 93, 631 (1995); D.H.Kobe and V.C.Aguilera-Navarro, Phys. Rev. A50, 933 (1994); N.Kumar, Pramana J.Phys. 25, 363 (1985); J.León, preprint quant-ph/9608013 (1996); D.Marolf, Phys. Rev. A50, 939 (1994); L.Mandelstamm and I.Tamm, J.Phys. 9, 249 (1945); J.G.Muga, S.Brouard and D.Macías, Ann. Phys. (N.Y.) 240, 351 (1995); J.G.Muga, J.P.Palao and C.R.Leavens, preprint quant-ph/9803087 (1987); J.G.Muga, R.Sala and J.P.Palao, preprint quant-ph/9801043, Superlattices and Microstructures 23 833 (1998); C.Piron, in Interpretation and Foundations of Quantum Theory, edited by H.Newmann (Bibliographisches Institute, Mannheim, 1979); M.Toller, preprint quant-ph/9805030 (1998); H.Salecker and E.P.Wigner, Phys. Rev. 109, 571 (1958); F.T.Smith, Phys. Rev. 118, 349 (1960); E.P.Wigner, Phys. Rev. 98, 145 (1955).

28. N.Yamada and S.Takagi, Prog.Theor.Phys. 85, 985 (1991); 86, 599 (1991); 87, 77 (1992); N. Yamada, Sci. Rep. Tôhoku Uni., Series 8, 12, 177 (1992); Phys. Rev. A54, 182 (1996); J.J.Halliwell and E.Zafiris, Phys.Rev. D57, 3351-3364 (1998); J.B.Hartle, Phys.Rev. D44, 3173 (1991); R.J.Micanek and J.B.Hartle, Phys.Rev. A54, 3795 (1996).

29. J.B.Hartle, Phys. Rev. D38, 2985 (1988). 
30. J.J.Halliwell, Phys. Rev. D48, 4785 (1993).

31. L.Schulman, Techniques and Applications of Path Integrals (Wiley, New York, 1981).

32. H.Goldstein, Classical Mechanics (Addison-Wesley, Reading MA, 1980).

33. J.S.Bell, Speakable and Unspeakable in Quantum Mechanics (Cambridge University Press, Cambridge, 1987).

34. A.Auerbach and S.Kivelson, Nucl. Phys. B257, 799 (1985).

35. J.B.Hartle and S.W.Hawking, Phys. Rev. 28, 2960 (1983).

36. S.W.Hawking, Phys. Rev. D32, 2489 (1985).

37. See, for example, the collection of articles in Physical Origins of Time Asymmetry, edited by J.J.Halliwell, J.Perez-Mercader and W.Zurek (Cambridge University Press, Cambridge, 1994). A recent discussion is K.Ridderbos, Studies in History and Philosophy of Modern Physics 30, 41(1999).

38. C.W.Gardiner, Quantum Noise (Springer-Verlag, Berlin, 1991).

39. J.Klauder, Ann. Phys. (NY) 254, 419 (1997), quant-ph/9604033) ; Nucl.Phys. B547,397, 1999, (hep-th/9901010); hep-th/0003297.

40. C.Kiefer, Phys. Rev. D38, 1761 (1988).

41. Y.Kazama and R.Nakayama, Phys. Rev. 32, 2500 (1985).

42. J.J.Halliwell, Prog.Theor.Phys. 102, 707 (1999). 